\title{
Epithelial and neutrophil interactions and coordinated response to Shigella in a human intestinal enteroid-neutrophil co-culture model
}

Jose M. Lemme-Dumit, ${ }^{1}$ Michele Doucet, ${ }^{2}$ Nicholas C. Zachos, ${ }^{2 *}$ and Marcela F. Pasetti ${ }^{1,3 *}$

${ }^{1}$ Department of Pediatrics, Center for Vaccine Development and Global Health, University of Maryland School of Medicine, Baltimore, MD, USA.

${ }^{2}$ Department of Medicine, Division of Gastroenterology and Hepatology, Johns Hopkins University School of Medicine, Baltimore, MD, USA.

${ }^{3}$ Lead contact

${ }^{*}$ Corresponding authors:

Marcela F. Pasetti, Ph.D.

685 West Baltimore St.; HSF-I Room 480

Baltimore, MD 21201

Email: mpasetti@som.umaryland.edu

Phone: 410-706-2341; Fax: 410-706-6205

Nicholas C. Zachos, Ph.D.

720 Rutland Avenue; Ross Research Building Room 943

Baltimore, MD 21205

Email: nzachos1@jhmi.edu

Phone: 410-614-0128; Fax: 410-955-9677 


\section{Abstract}

2 Polymorphonuclear neutrophils $(\mathrm{PMN})$ are recruited to the gastrointestinal mucosa in response

3 to infection. Herein, we report the development and the characterization of an ex vivo tissue co-

4 culture model consisting of human intestinal enteroid monolayers and PMN, and a mechanistic

5 interrogation of PMN-epithelial cell interaction and response to Shigella, a primary cause of

6 childhood dysentery. Co-culture integration, barrier function, PMN phenotypic and functional

7 attributes, and innate immune responses were examined. PMN within the enteroid monolayers

8 acquired a distinct activated/migratory phenotype that was influenced by direct epithelial cell

9 contact as well as by molecular signals. Seeded on the basal side of the intestinal monolayer,

10 PMN intercalated within the epithelial cells and moved paracellularly toward the apical side. Co-

11 cultured PMN also increased basal secretion of IL-8. Shigella added to the apical surface of the

12 monolayers evoked additional PMN phenotypic adaptations, including increased expression of

13 cell surface markers associated with chemotaxis and cell degranulation (CD47, CD66b and

14 CD88). Shigella infection triggered rapid transmigration of PMN to the luminal side of

15 monolayers resulting in phagocytosis and killing of organisms. Shigella infection modulated

16 cytokine production in the co-culture; apical MCP-1, TNF- $\alpha$, and basolateral IL-8 production

17 were downregulated, while basolateral IL-6 secretion was increased. We demonstrate, for the

18 first time, PMN phenotypic adaptation, mobilization, and coordinated epithelial cell-PMN innate

19 response upon Shigella infection in the human intestinal environment. The epithelial cell-PMN

20 co-culture represents a technical innovation for mechanistic interrogation of gastrointestinal

21 physiology, host-microbe interaction, innate immunity, and evaluation of preventive/therapeutic

22 tools.

\section{Importance}

25 Studies of mucosal immunity and microbial host cell interaction have traditionally relied on

26 animal models and in vitro tissue culture using immortalized cancer cell lines, which render non- 
27 physiological and often unreliable results. Herein we report the development and 28 characterization of an ex vivo enteroid-PMN co-culture consisting of normal human intestinal

29 epithelium and a mechanistic interrogation of PMN and epithelial cell interaction and function in

30 the context of Shigella infection. We demonstrated tissue-driven phenotypic and functional

31 adaptation of PMN and a coordinated epithelial cell and PMN response to Shigella, a primary

32 cause of dysentery in young children in the developing world.

33

34 


\section{Introduction}

36 The intestinal epithelium creates a physical and molecular barrier that protects the host from

37 potentially damaging elements in the constantly changing outside environment. Epithelial barrier

38 function is supported by a diverse population of underlying immune cells, which deploy a variety

39 of host-defense mechanisms against harmful agents (1). Coordinated signals resulting from

40 microbial sensing, cell-to-cell contact, cytokines, and other chemical mediators determine the

41 type and extent of responses of gut immune cells, balancing tissue homeostasis with effective

42 anti-microbial function via inflammation.

43 Advances in understanding intestinal physiology, pathophysiology, and host immunity have

44 traditionally relied on studies conducted in animal models (or animal tissue) and in traditional

45 tissue culture systems using colon cancer cell lines. Animal models, including the use of mutant

46 mouse strains, have contributed to the mechanistic understanding of the composition, function,

47 regulatory processes, and operatives of immunity at the gut mucosa. Unfortunately, host

48 restrictions limit the utility and value of animal models $(2,3)$. This is the case for many enteric

49 pathogens for which small animals fail to recreate disease as it occurs in humans. Likewise,

50 immortalized (transformed) cell lines (e.g., HT-29, Caco-2, and T84) do not reflect human

51 physiological responses but rather the aberrant behavior of diseased cells (e.g., karyotype

52 defects). These cell-line based cultures also lack the multicellular complexity of the human

53 intestinal epithelium, which further reduces the reliability and significance of the data generated.

54 The establishment of human enteroids from Lgr5 $^{+}$intestinal stem cells was a breakthrough in

55 tissue culture technology (4). Since then, three-dimensional (3D) intestinal enteroids have been

56 widely used as models to study human gut physiology and pathophysiology as well as host-

57 microbe interactions (5-7). Not only do enteroids render a truer physiological representation of

58 the human epithelium, but they also offer a practical and reliable system to probe mechanisms

59 and interventions at the gut mucosal interface. The 3D spheroid conformation can be adapted to

60 produce a 2D monolayer configuration with enteroids seeded on a semipermeable membrane 
61 (i.e., Transwell insert) (8-10). An important practical advantage to this simplified format is that it

62 allows for direct and controlled access to the apical (mimicking the lumen) and basolateral side

63 of the epithelial cells, thus facilitating experimental manipulation and evaluation of outcomes.

64 Enteroid monolayers, which can be generated from any gut segment, can mimic the 65 undifferentiated (crypt-like) and differentiated (villus-like) profile of the intestinal epithelial cells 66 (i.e., absorptive enterocytes, goblet cells, enteroendocrine cells, and Paneth cells) (11) and 67 display segment-specific phenotypic and functional attributes of the normal human gut (12). To 68 better recreate the cellular complexity of the gastrointestinal mucosal barrier, we devised a 69 human primary cell co-culture system consisting of enteroid monolayers and macrophages 70 seeded on the basolateral side (11). Studies using this enteroid-macrophage co-culture model 71 demonstrated physical and cytokine/chemokine-mediated interactions between intestinal

72 epithelial cells and macrophages in the presence of pathogenic E. coli $(11,13)$. Aiming to 73 expand this co-culture conformation to include other phagocytic cells, we established an ex vivo 74 co-culture model containing intestinal epithelial cells and human primary polymorphonuclear 75 neutrophils (PMN) facing the monolayers' basal membrane. The histological and functional 76 features of this co-culture model, such as cell integration, PMN phenotype, PMN-epithelial cell 77 physical and molecular interactions, and cell function were characterized. Coordinated epithelial 78 and PMN anti-microbial response was examined using Shigella as model enteric pathogen. 79 Shigella causes diarrhea and dysentery in humans by trespassing the colonic barrier via M cells 80 and infecting epithelial cells, and this process involves massive recruitment of PMN (14). The 81 enteroid-PMN co-culture modeled the paradoxical role of PMN contributing to inflammation and 82 controlling infection.

\section{Results}

85 Establishment of a PMN-enteroid co-culture and PMN-epithelial cell interaction. To 86 interrogate PMN adaptation and function in the human gut, we established an enteroid-PMN co- 
87 culture model that contains human enteroid monolayers and peripheral blood isolated human

88 PMN. A human enteroid-macrophage co-culture with similar configuration has been developed

89 by our group (11). Human ileal 3D organoids derived from Lgr5 ${ }^{+}$-containing biopsies from

90 healthy subjects were seeded upon the inner (upper) surface of a Transwell insert and allowed

91 to grow until they reached confluency. PMN isolated from peripheral blood of healthy human

92 adult volunteers and exhibiting a CD15 ${ }^{+} \mathrm{CD} 16^{+} \mathrm{CD} 14^{-}$phenotype (15) were seeded on the outer

93 (bottom) surface of the insert (Figure 1A). Confocal immunofluorescence microscopy and H\&E

94 staining confirmed the co-culture's expected epithelial cell polarity with the brush border

95 oriented towards the luminal side (apical compartment) and adherent PMN facing the

96 basolateral side of the monolayer (Figure 1B). Interestingly, PMN rapidly mobilized from the

97 bottom side of the insert (where they were seeded) towards the epithelium. Within $2 \mathrm{~h}$ of being

98 added to the monolayers, PMN migrated from the insert and through the insert's pores (Figure

99 S1) and intercalated within the epithelial cells (Figures 1B, C). The migrating PMN could be

100 retrieved and enumerated in the apical compartment media (Figure 1D). The addition of PMN to

101 the enteroid monolayer increased epithelial permeability, as shown by reduction in

102 transepithelial electrical resistance (TER); although the difference did not reach statistical

103 significance (Figure 1E). Because human IL-8 promotes PMN recruitment (16), we examined

104 the effect of exogenous IL-8 on the mobilization of PMN co-cultured with epithelial cells and on

105 monolayer permeability. Apical treatment of monolayers with $100 \mathrm{ng} / \mathrm{ml} \mathrm{IL-8}$ increased PMN

106 epithelial transmigration (1.8-fold) (Figure 1D) and barrier permeability; the PMN-induced

107 decrease in TER became statistically significant in the presence of IL-8 (Figure 1F). Importantly,

108 IL-8 alone did not affect the permeability of monolayers (Figure 1F).

109 Since cell movement is influenced by molecular mediators, we next examined the presence of

110 cytokines (pro- and anti-inflammatory) and chemoattractant molecules in tissue culture media

111 collected from the apical and basolateral compartments of enteroid monolayers alone and of

112 enteroid-PMN co-cultures. Basolateral levels of IL-8 produced by the PMN-containing enteroids 
113 were 10 -fold higher as compared to IL-8 produced by the monolayers alone, while MCP-1 and

114 TNF- $\alpha$ remained unaffected by addition of PMN (Figure 1G). Similarly, the presence of PMN did

115 not affect apical secretion of MCP-1, TNF- $\alpha$, and IL-8 (Figure 1G). Production of MCP-1 was

116 distinctly polarized, with higher levels being released to the apical side of the epithelial barrier.

$117 \mathrm{IL}-1 \beta, \mathrm{IL}-6, \mathrm{IL}-10, \mathrm{IL}-12 \mathrm{p} 70, \mathrm{IFN}-\gamma$, and TGF- $\beta 1$ were measured but determined to be below the

118 limit of detection of the assay for each cytokine.

119 Taken together, these results demonstrate adequate engraftment of PMN on the basolateral

120 side of the enteroid monolayer, rapid migration of PMN across the monolayer to the luminal

121 side, PMN-induced basolateral secretion of IL-8, and membrane de-stabilization (increased

122 permeability) by IL-8-enhanced PMN transepithelial movement.

124 The human intestinal epithelium environment determines PMN immune phenotype and

125 functional capacity. Cell phenotype, morphology, and function can be affected by the

126 surrounding tissue and molecular environment. We hypothesized that the immune phenotype

127 and functional capacity of PMN added to the enteroid monolayer would be influenced by their

128 proximity or direct contact with the intestinal epithelium. To explore this hypothesis, we

129 determined the expression of cell surface markers and phenotypic features of PMN isolated

130 from peripheral blood in comparison with those of PMN within the enteroid co-culture. Two

131 populations of co-cultured PMN were investigated following $2 \mathrm{~h}$ of incubation: PMN residing

132 within the monolayer and in direct contact with the epithelial cells and PMN harvested from the

133 basolateral media as tissue adjacent milieu (Figure 2A). PMN co-cultured with enteroid

134 monolayers had a distinct phenotypic profile as compared with PMN obtained from peripheral

135 blood. Regardless of their location, PMN co-cultured with enteroid monolayers exhibited

136 increased expression of CD18 ( $\beta 2$ integrin), a molecule that participates in extravasation of

137 circulating PMN, as well as upregulation of CD47, a receptor for membrane integrins involved in 
138 cell adhesion and migration, and of CD88 (C5a receptor), a molecule that mediates chemotaxis, 139 granule enzyme release, and super anion production (Figure 2B; Figure S2B). CD66b, a marker 140 of secondary granule exocytosis was likewise increased, but only in PMN harvested from the 141 basolateral media (not in contact with cells) (Figure 2C; Figure S2B). In contrast, expression of 142 CD182 (CXCR2 or IL-8RB) was reduced in all PMN added to co-culture, without distinction of 143 retrieving site (Figure 2C; Figure S2B). PMN residing in close contact with epithelial cells 144 exhibited increased expression of CD15 (E-selectin), a molecule that mediates PMN 145 extravasation; CD16 (FcyRIII), a receptor for lgG that mediates degranulation, phagocytosis, 146 and oxidative burst; and CD11b ( $\alpha$ integrin), a protein that facilitates PMN adhesion and, along 147 with CD18, forms the Mac-1 complex implicated in multiple anti-microbial functions (e.g., 148 phagocytosis, cell-mediated cytotoxicity, cellular activation) (Figure 2D; Figure S2B). These 149 results demonstrate that PMN within the intestinal epithelial environment undergo unique 150 phenotypic adaptations, some of which are driven by molecular signals while others require 151 direct PMN-epithelial cell contact.

153 PMN interaction with Shigella as a model enteric pathogen. To interrogate human epithelial

154 cell and PMN interactions in the context of an enteric infection, we exposed the co-culture to 155 wild type Shigella flexneri 2a (strain 2457T) as a model pathogen. PMN participate in Shigella 156 pathogenesis through secretion of pro-inflammatory cytokines and deploy anti-microbial 157 functions including phagocytosis, proteolytic enzymes, anti-microbial proteins, and neutrophil 158 extracellular traps (NETs) production. We first determined baseline responses of peripheral 159 blood PMN in the presence of Shigella. PMN phagocytosed FITC-labeled S. flexneri 2a 2457T 160 within 10 min of exposure; bacterial phagocytosis increased over time (up to $1 \mathrm{~h}$ tested) reaching 161 a maximum effect at $30 \mathrm{~min}$ (Figure $3 \mathrm{~A}$ ). In parallel, the number of bacteria recovered from the 162 culture supernatant of Shigella-exposed PMN decreased significantly within 30- and 60-min 
163 exposure, in comparison to the number of bacteria recovered from control wells containing

164 Shigella alone (in the absence of PMN) resuspended in tissue culture media or media that had

165 been exposed to PMN to control for any soluble bactericidal source (Figure 3B). PMN viability

166 was not affected during the first $2 \mathrm{~h}$ of Shigella exposure, but decreased significantly by $3 \mathrm{~h}$ post

167 infection (Figure 3C). Shigella-exposed PMN exhibited changes in cell morphology and motility;

168 formation of pseudopodia (projections of the cell membrane that enable locomotion) was

169 observed within 30 min post infection (Figure 3D). In the presence of Shigella, PMN also

170 displayed dynamic amoeboid motility toward the bacteria, and released NETs evidenced by a

171 thick, diffuse, and opaque weave of DNA and entrapped bacilli (Figure 3E). FITC-stained

172 Shigella colocalized with PMN phagolysosome (Figure 3F). In addition, PMN upregulated

173 production and secretion of IL-8 and IL-1 $\beta$, which are key molecular mediators of Shigella

174 pathogenesis, $2 \mathrm{~h}$ post infection, while production of IL-6 and MCP-1 was downregulated;

175 production of TNF- $\alpha$ was not affected (Figure $3 \mathrm{G}$ ). IL-10, TGF- $\beta 1$, and IFN- $\gamma$ were also

176 measured but were below the limit of detection of the assay. A principal component analysis

177 (PCA) of PMN function, viability, and cytokine production revealed an association between

178 PMN-mediated phagocytosis and bactericidal activity with IL-8, IL-1 $\beta$, and TNF- $\alpha$ production as

179 indicated by principal component 1 on a PCA plot (Figure $3 \mathrm{H})$. These results showed that PMN

180 anti-microbial responses against Shigella involved morphological changes, phagocytic activity,

181 and modulation of inflammatory cytokines.

183 Coordinated epithelial cell and PMN responses to Shigella in the co-culture model. We

184 interrogated PMN and epithelial cell interactions and coordinated responses to Shigella in the

185 PMN-enteroid co-cultures (Figure 1A). PMN were added to the enteroid monolayer as described

186 above, allowed to settle for $2 \mathrm{~h}$, and then apically exposed to wild type $S$. flexneri $2 \mathrm{a}(\mathrm{MOI}=10)$

187 for another 2h. Non-exposed co-cultures served as controls. Consistent with our previous 
188 observation, PMN facing the basolateral side of the epithelial cells moved swiftly through the

189 filter pores, traversed between the epithelial cells and across the monolayer, and protruded on

190 the apical side. PMN basolateral-apical transmigration increased in the presence of Shigella

191 (Figure 4A). PMN that traversed across the monolayer phagocytosed bacteria on the luminal

192 surface as evidenced by confocal fluorescent microscopy (Figure 4B; CD16 ${ }^{+}$PMN are stained in

193 green, engulfed S. flexneri 2 a in red; actin in white). The process created a hole in the otherwise

194 confluent epithelium (Figure 4C). Addition of PMN to the enteroid monolayers enabled Shigella

195 to reach the basolateral side, whereas in the absence of PMN, Shigella was unable to trespass

196 the intact enteroid (Figure 4D). Reduced (1.3-fold) TER values were observed in the Shigella-

197 exposed PMN-enteroid co-culture as compared with Shigella-exposed monolayers alone or the

198 monolayer control (no PMN, no Shigella) (Figure 4E), consistent with loss of barrier function and

199 tissue damage that allowed bacterial translocation.

200 We next examined cytokines produced by Shigella-infected and non-infected PMN-enteroid co-

201 cultures. MCP-1, TNF- $\alpha$, and IL-8 were detected in the apical and basolateral compartments, as

202 described above. Shigella infection resulted in reduced apical production of MCP-1 and TNF- $\alpha$

203 by the co-cultured cells as well as in increased production of IL-6 and substantially reduced

204 secretion of IL-8 basolaterally (Figure 4F). IL-6 was only detected in the infected cultures and

205 exhibited a clear basolateral polarization (Figure 4F). Cultures were also tested for presence of

206 IL-1 $\beta$, which along with IL-8, are hallmarks of Shigella pathogenesis, but were not detectable.

207 Collectively, these observations demonstrate that Shigella infection causes active recruitment of

208 PMN to the luminal side, which results in discernible damage of the enteroid monolayer that

209 paradoxically enables Shigella penetration and basolateral infection; still PMN actively engulfed

210 bacteria and increased production and secretion of inflammatory cytokines to the apical and

211 basolateral sides, respectively. 
213 Phenotypic changes in PMN co-cultured with epithelial cells in response to Shigella

214 infection. Additional experiments were conducted to determine the phenotypic features of PMN

215 co-cultured with epithelial cells upon Shigella infection. PMN co-cultured with intestinal epithelial

216 cells and exposed to Shigella exhibited increased expression of CD88, CD47, and CD66b as

217 compared with PMN in co-cultures that remained uninfected (Figure 5A; Figure S3). In contrast,

218 CD15 and CD18 expression was decreased on PMN from Shigella-infected co-cultures (Figure

219 5B; Figure S3). CD16, CD11b, and CD182 remained unchanged (Figure 5C; Figure S3). These

220 results suggest that PMN residing within the intestinal environment undergo immune phenotypic

221 adaptation as a result of pathogen exposure consistent with increased anti-microbial function

222 (i.e., activation and expression of molecules that facilitate chemotaxis and epithelial

223 transmigration).

Discussion

Epithelial cells and innate phagocytic cells underlying the intestinal epithelium work

227 synergistically, preventing the trespassing of harmful agents and deploying rapid and effective

228 host defense mechanisms against pathogens. PMN are the first innate immune cells recruited in

229 response to gastrointestinal tissue inflammation and infection and they play a critical role

230 initiating host immune responses (17). Patients with neutrophil disorders are prone to recurrent

231 microbial infection $(18,19)$. We report in this manuscript the successful establishment of an ex

232 vivo primary human intestinal epithelial-PMN co-culture and results from a mechanistic

233 interrogation of cell interactions and innate responses to Shigella as a relevant diarrhoeagenic

234 pathogen.

235 Our group developed the first ex vivo human enteroid- and monocyte-derived

236 macrophage co-culture model in a monolayer format (11). The same approach was used to

237 produce the PMN-enteroid co-culture described herein. Differently from macrophages, which

238 remained in the basolateral side of the monolayer (where seeded) and responded to luminal 
organisms by extending transepithelial projections between adjacent epithelial cells, PMN

240 rapidly migrated from the basolateral side of the epithelial cell and across the monolayer via the

241 paracellular space. Histological and confocal microscopy images revealed PMN crawling

242 through the Transwell insert pores, embedding at the base of the epithelial cells, and emerging

243 on the luminal side of the enteroid monolayer, all within a few hours of co-culture. While

244 macrophages contributed to cell differentiation and stabilized the epithelial barrier in our

245 previous studies, PMN transmigration resulted in increased barrier permeability that enabled

246 bacteria invasion. Intestinal epithelial repair events (e.g., cell proliferation and migration, and

247 closure of leaking epithelial lateral spaces) reportedly begin minutes after acute mucosal barrier

248 injury (20). As a corollary of these observations, we are investigating epithelial repair

249 subsequent to PMN-induced inflammation and cell disruption, and the mechanisms and

250 elements involved.

251 The capacity of PMN to migrate across the vascular endothelium (21) and a variety of

252 tissues $(22,23)$, including epithelial cell layers (24-27) has been documented in vivo (mainly in

253 animal models) or in vitro using cell lines. These processes have been associated with PMN

254 activation as a result of microbial sensing, inflammation, or danger signals. The level of

255 myeloperoxidase (MPO), one of the principal enzymes contained in PMN granules and released

256 upon activation, in stool is, in fact, a biomarker of inflammatory bowel disease severity (28). In

257 our human PMN and intestinal epithelial cell co-culture model, PMN migrated even in the

258 absence of external stimulatory signals. PMN are not typically present in the homeostatic gut

259 but actively recruited by signs of inflammation or infection (29); therefore, unprovoked PMN

260 migration would not be expected. From the technical standpoint, the $3.0 \mu \mathrm{m}$ pore size Transwell

261 membrane used in our model allowed not only for successful and consistent growth of enteroid

262 monolayers, but also the basolateral-to-apical migration of PMN; this system is more practical

263 than the traditional growing of epithelial cells in an inverted manner, typically used for cancer

264 cell lines. 
The tissue microenvironment can influence immune cell phenotype and effector function capabilities $(30,31)$. PMN from peripheral blood exhibited rapid phenotypic changes when

267 incubated with enteroid monolayers. They acquired an activated phenotype that was triggered

268 by tissue-derived signals. Compared to the PMN from peripheral blood, PMN co-cultured with

269 epithelial cells had increased expression of CD18, which favors cell binding, of CD88, which is

270 the receptor for $\mathrm{C5a}$ and facilitates degranulation and chemotaxis, and of CD47, a cell surface

271 glycoprotein that supports transmigration across endothelial and epithelial cells (32-34). PMN

272 phenotypic changes were influenced by their spatial location, i.e., whether they were in direct

273 contact with epithelial cells or simply present in basolateral culture media. PMN in close

274 proximity with the epithelium (the migratory PMN) had upregulated expression of CD15, which

275 participates in chemotaxis and extravasation from circulation, as well as FcyRIII (CD16), a low

276 affinity Fc receptor for $\operatorname{lgG}$, and CD11b, a marker of cell adhesion and anti-microbial function

277 (phagocytosis, degranulation, oxidative burst) $(35,36)$. On the other hand, PMN retrieved from

278 the basolateral media, and which had not been in contact with the epithelial layer, exhibited

279 increased expression of CD66b, indicative of PMN activation and degranulation (37). This is to

280 our knowledge the first detailed description of dynamic changes of PMN immune phenotype in a

281 translationally relevant model of the human intestinal epithelium.

282 MCP-1/CCL2, a chemoattractant and enhancer of bacterial killing and survival of

283 phagocytic cells and IL-8, a potent promoter of PMN migration and tissue infiltration (38, 39),

284 were abundantly produced by the ileal monolayers in our co-culture model. TNF- $\alpha$, a recruiter

285 and activator of phagocytic cells (40) was also detected, albeit at lower levels. IL-8, a hallmark

286 product of intestinal epithelial cells, was further sourced by the PMN within the co-culture and

287 released to the basolateral media; in vivo this subepithelial surge of IL-8 excess likely

288 contributes to PMN recruitment. Expression of MCP-1, IL-8, and TNF- $\alpha$ has been reported in

289 intestinal tissue of healthy adults in steady state $(41,42)$. 
Again, when comparing PMN- and macrophage-enteroid co-cultures in terms of cytokine

291 production, clear differences in innate immune functions emerged; while macrophages

292 contributed to high levels of IFN- $\gamma$ and IL-6, these cytokines were not detected in co-cultures

293 containing PMN. The models were therefore capable of discerning morphological as well as

294 functional cell adaptation.

Shigella invades the human colon and rectal mucosa and causes severe inflammation, massive recruitment of PMN, and tissue destruction (14). Bloody/mucous diarrhea (dysentery) with large numbers of PMN in stool are hallmarks of shigellosis (43). lipopolysaccharide trancytosed to the basolateral side of T84 cells in the presence of human normal sera and enhanced adherence of subepithelial PMN through IL-8 signaling (48).

PMN transmigration across the intestinal monolayer in our co-culture model caused

307 microscopically visible epithelial cell damage and created a conduit for bacterial invasion and 308 possibly amplification of infection. This observation is consistent with focal breakdown of the 309 epithelial cell surface attributed to PMN migration in various disease states including infectious

310 enterocolitis (49). Our findings challenge for the first time the notion that $\mathrm{M}$ cells are necessary

311 for Shigella epithelial translocation and invasion, and hint that the infiltration and barrier

312 disruption by PMN offer another mechanism by which Shigella and other invasive enteric 313 pathogens access the host internal compartment. We are presently studying reverse

314 transmigration of bacteria-loaded PMN (out of the lumen and back to the basolateral side) as a 315 possible means to initiate adaptive immunity through cross-presentation. The model lends itself 
316 to study of molecules that reduce PMN activity in the context of enteric infections and could 317 prevent intestinal injury and inflammation.

318 Although acting in a "brute force" manner, PMN deployed potent anti-microbial activity

319 against Shigella. As expected, PMN from circulation phagocytosed Shigella rapidly and 320 efficiently and entrapped bacteria into NET structures. PMN anti-microbial functions coincided 321 with increased production of IL-8 and the pyroptosis-inducer IL-1 $\beta$, and downregulation of IL-6

322 and MCP-1. Likewise, PMN embedded within the epithelial cells promptly mobilized upon 323 sensing Shigella on the epithelial cell surface and exhibited robust phagocytic capacity.

324 Intriguingly, IL-8 levels were reduced, IL-1 $\beta$ was absent, and IL- 6 was increased in the infected 325 PMN-epithelial co-culture as compared to non-infected. A reduction of IL-8 production had been 326 reported in Shigella-infected human colonic explants, which was ascribed to anti-inflammatory 327 bacterial proteins or death of IL-8 secreting cells (50). Reduced levels of these inflammatory 328 cytokines may also reflect a regulatory feedback to prevent further tissue damage. Heightened 329 levels of IL-6 and reduced TNF- $\alpha$ during infection may suggest a protective epithelial 330 mechanism after injury $(51,52)$. In addition, IL- 6 has been attributed a beneficial role in 331 enhancing Th17-protective immunity against Shigella re-infection (53). Because cytokines 332 measured in the co-culture supernatant represent the total amount produced by diverse cell 333 types, this readout is limited in its capacity to discern subtle differences between culture 334 conditions.

335 The immune phenotype of PMN in the co-culture adapted again as a result of Shigella 336 infection, with further increases in activation/granule-associated markers CD66b, CD88, and 337 CD47. CD47 has been implicated in PMN paracellular migration through epithelial cells in 338 response to bacterium-derived leukocyte chemoattractant $\mathrm{N}$-formyl-methionyl-leucyl339 phenylalanine, in a process that involves intracellular distribution and increased CD47 cell 340 surface expression (33). CD47-deficient mice have increased susceptibility to E. coli as a result 
341 of reduced PMN trafficking and bacterial killing activity (54). This finding is consistent with our

342 observed upregulation of CD47 in Shigella-exposed PMN, which is likely associated with PMN's

343 anti-microbial activity. CD16 and CD11b expression were unaltered on PMN co-cultured with

344 intestinal enteroids and exposed to Shigella, indicating a preserved phagocytic capacity,

345 whereas extravasation and cell adhesion markers CD15 and CD18 were downregulated. It has

346 been reported that CD47 expression increases gradually and modulates CD11b-integrin

347 function and CD11b/CD18 surface expression on PMN, suggesting a regulatory mechanism

348 between these molecules $(33,55)$. Expression of CD47 is self-protective; it avoids clearance by

349 phagocytic cells (56). The exact role of CD47 expression on PMN during Shigella infection

350 remains to be elucidated.

351 Human intestinal xenografts in immunodeficient mice have been used to model

352 interactions of Shigella with the human intestine in vivo. The model failed to discern any role of

353 PMN in ameliorating or exacerbating disease, but revealed larger intracellular bacteria in PMN-

354 depleted mice; the authors conclude that while PMN may contribute to tissue damage, they are

355 important in controlling bacteria dissemination. The combination of species, immunodeficient

356 background, and impracticality are major confounders/limitations of this model (57).

357 Our studies provided new insights on the close communication between PMN and

358 epithelial cells, phenotypic and functional changes on PMN prompted by the gastrointestinal

359 environment, and their coordinated responses to Shigella as a model gastrointestinal pathogen.

360 The PMN-epithelial cell co-culture described here provides a translationally relevant ex vivo 361 model to study human epithelial cell-PMN physiology and pathophysiology, as well as host cell

362 interactions and innate responses to enteric organisms. This model could be useful to

363 interrogate innate immune defense mechanisms to enteric pathogens and to support the 364 development and evaluation of preventive or therapeutic tools. 


\section{Human PMN isolation}

368 Human peripheral blood was collected in EDTA tubes (BD Vacutainer) from healthy volunteers

369 enrolled in University of Maryland Institutional Review Board (IRB) approved protocol \#HP-

370 40025-CVD4000, and methods were conducted in compliance with approved Environmental

371 Health and Safety guidelines (IBC \#00003017). PMN were obtained by Ficoll-Paque (PREMIUM

372 solution, GE Healthcare Bio-Sciences AB, Sweden) gradient centrifugation following dextran

373 (Alfa Aesar, USA) sedimentation (58). Contaminating erythrocytes were removed by hypotonic

374 lysis. After washing, cells (>95\% of PMN determined by flow cytometry and May-Grünwald-

375 Giemsa stained cytopreps) were suspended in enteroid differentiation media (DFM) without

376 antibiotics and immediately used. Cell counting and viability were determined using Guava

377 Viacount Flex Reagent (Luminex, USA) following the manufacturer's instruction and analyzed in

378 Guava 8HT using Viacount software (Luminex, USA).

\section{Tissue collection and enteroid generation}

381 Human enteroid cultures were established from biopsy tissue obtained after endoscopic or

382 surgical resection from healthy subjects at Johns Hopkins University under Johns Hopkins

383 University IRB approved protocol \#NA-00038329, as previously described (59). Briefly,

384 enteroids generated from isolated intestinal crypts from ileal segments were maintained as cysts

385 embedded in Matrigel (Corning, USA) in 24-well plates and cultured in Wnt3A containing non-

386 differentiated media (NDM) (58). Multiple enteroid cultures were harvested with Culturex

387 Organoid Harvesting Solution (Trevigen, USA), and small enteroid fragments were obtained by

388 digestion with TrypLE Express (ThermoFisher) in $37^{\circ} \mathrm{C}$ water bath for 90 seconds. Enteroid

389 fragments were resuspended in NDM containing $10 \mu \mathrm{M}$ Y-27632 and $10 \mu \mathrm{M}$ CHIR 99021

390 inhibitors (Tocris) (NDM+inhibitors). The inner surface of Transwell inserts (3.0 $\mu \mathrm{m}$ pore

391 transparent polyester membrane) pre-coated with $100 \mu \mathrm{l}$ of human collagen IV solution (34 
$392 \mu \mathrm{g} / \mathrm{ml}$; Sigma-Aldrich, USA) were seeded with $100 \mu \mathrm{l}$ of an enteroid fragment suspension, and

$393600 \mu \mathrm{l}$ of NDM+inhibitors was added to the wells of a 24-well tissue culture plate and incubated

394 at $37^{\circ} \mathrm{C}, 5 \% \mathrm{CO}_{2}$, as previously described (58). NDM without inhibitors were replaced after $48 \mathrm{~h}$,

395 and fresh NDM was added every other day; under these conditions, enteroid cultures reached

396 confluency in 14-16 days. Monolayer differentiation was induced by incubation in Wnt3A-free

397 and Rspo-1-free DFM without antibiotics for 5 days (11). Monolayer confluency was monitored

398 by measuring TER values with an epithelial voltohmmeter (EVOM ${ }^{2}$; World Precision

399 Instruments, USA). The unit area resistances $\left(\mathrm{ohm}^{*} \mathrm{~cm}^{2}\right)$ were calculated according to the

400 growth surface area of the inserts $\left(0.33 \mathrm{~cm}^{2}\right)$.

402 PMN-enteroid co-culture

403 Differentiated enteroid monolayers seeded on Transwell inserts were inverted and placed into 404 an empty 12 -well plate. PMN $\left(5 \times 10^{5}\right.$ in $50 \mu$ l of DFM) were added onto the bottom surface of the 405 inserts and cells were allowed to attach for $2 \mathrm{~h}$ at $37^{\circ} \mathrm{C}, 5 \% \mathrm{CO}_{2}$ (inserts remained wet 406 throughout this process). The inserts were then turned back to their original position into a 24407 well plate, and DFM was added to the insert $(100 \mu \mathrm{l})$ and into the well $(600 \mu \mathrm{l})$. Approximately $40845 \%$ of the total PMN remained attached to the Transwell insert. TER measurements were 409 collected after $2 \mathrm{~h}$, allowing monolayer recovering.

\section{Shigella flexneri 2 a infection}

412 Shigella flexneri 2a wild type strain $2457 \mathrm{~T}$ was grown from frozen stocks $\left(-80^{\circ} \mathrm{C}\right)$ on Tryptic Soy 413 Agar (TSA) (Difco BD, USA) supplemented with $0.01 \%$ Congo Red dye (Sigma-Aldrich)

414 overnight at $37^{\circ} \mathrm{C}$. Bacterial inoculum was made by resuspending single red colonies in sterile

415 1X PBS pH 7.4 (Quality Biological). Bacterial suspension was adjusted to the desired 416 concentration ( $\left.1 \times 10^{8} \mathrm{CFU} / \mathrm{ml}\right)$ in advanced DMEM/F12 without serum. A bacterial suspension 
417 containing $\sim 5 \times 10^{6} \mathrm{CFU}$ in $50 \mu \mathrm{l}$ was added directly to PMN (for 30-60 min) or to the apical

418 compartment of enteroid monolayers alone or PMN-enteroid co-culture (for $2 \mathrm{~h}$ ), a multiplicity of

419 infection of 10 relative to 1 PMN.

\section{$421 \quad$ PMN transmigration}

422 Basolateral-to-apical PMN transmigration was quantified by measurement of PMN MPO using a 423 commercial kit (Cayman Chemical, Ann Arbor, MI) as previously described (60). The assay was 424 standardized with a known number of human PMN. MPO activity in lysates of enteroid 425 monolayer alone was negligible.

\section{PMN phagocytosis}

428 S. flexneri 2 a cultures grown overnight as described above were washed, resuspended in sterile

429 PBS and incubated with FITC (Sigma-Aldrich) $(20 \mu \mathrm{g} / \mathrm{ml})$ for $30 \mathrm{~min}$ at $37 \square \mathrm{C}$. The bacteria 430 suspension was thoroughly washed and adjusted to $\sim 10^{8} \mathrm{CFU} / \mathrm{ml}$ in sterile PBS/glycerol (1:2), 431 and stored at $-80 \square \mathrm{C}$ until used. The day of the assay, FITC-labeled Shigella was incubated with $432 \mathrm{PMN}$-autologous human sera for $30 \mathrm{~min}$ at $37 \square \mathrm{C}$. Opsonized bacteria $5 \times 10^{6} \mathrm{CFU}$ was added to

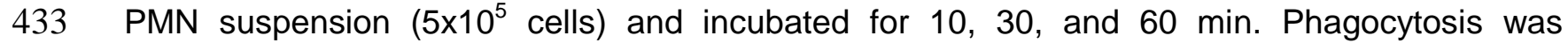
434 measured by flow cytometry. External fluorescence was blocked with the addition of trypan blue, 435 and the difference between MFI blocked and non-blocked samples was used to calculate \% 436 phagocytosis $(61,62)$.

\section{H\&E and immunofluorescence staining}

439 PMN-enteroid co-culture cells were fixed in aqueous 4\% paraformaldehyde (PFA; Electron 440 Microscopy Sciences, USA) at room temperature (RT) for 45 min and then washed with PBS. 441 For H\&E staining, monolayers were kept for at least $48 \mathrm{~h}$ in formaldehyde solution, then 
442 embedded in paraffin, sectioned, mounted on slides, and stained with H\&E. For

443 immunofluorescence, cells were permeabilized and blocked for 30 min at RT in PBS containing

$44415 \%$ FBS, 2\% BSA, and $0.1 \%$ saponin (all Sigma-Aldrich, USA). Cells were rinsed with PBS

445 and incubated overnight at $4 \square \mathrm{C}$ with primary antibodies: mouse anti-CD16 (LSBio, USA), rabbit

446 anti-S. flexneri 2a (Abcam, USA) diluted 1:100 in PBS containing 15\% FBS and $2 \%$ BSA.

447 Stained cells were washed with PBS and incubated with secondary antibodies: goat anti-mouse

448 AF488, goat anti-rabbit AF594 (all Thermo Fisher Scientific, USA) diluted 1:100 in PBS 1h at

449 RT; phalloidin AF594 or AF633 (Molecular Probes, Thermo Fisher Scientific) was included in

450 this step for actin visualization. Cells were washed and mounted in ProLong Gold Antifade

451 Reagent with DAPI (Cell Signaling Technology, USA) for nuclear staining and maintained at

$4524 \square$ C. Lysosome was stained with LysoTracker ${ }^{T M}$ Red DND-99 (Thermo Fischer Scientific)

453 following the manufacturer's instructions.

455 Immunofluorescence microscopy

456 Confocal imaging was carried out at the Confocal Microscopy Facility of the University of

457 Maryland School of Medicine using a Nikon W1 spinning disk confocal microscope running NIS-

458 Elements imaging software (Nikon). Images were captured with a 40X or 60X oil objective and

459 settings were adjusted to optimize signal. Immunofluorescence imaging (Figure 3F) was carried

460 out using EVOS FL Imaging systems (fluorescent microscope) at 40X objective lens. Images

461 were collated using FIJI/ImageJ $(\mathrm{NIH})$. Signal processing was applied equally across the entire

462 image. Actin channel was arranged to white-grey color for contrast purpose.

\section{Flow cytometry}

465 Cell phenotype was determined using the following human specific monoclonal antibodies from

466 BD Pharmingen: HI98 (anti-CD15, FITC-conjugated), M5E2 (anti-CD14, APC-conjugated), 3G8

467 (anti-CD16, PE/Cy7-conjugated), D53-1473 (anti-CD88, BV421-conjugated), and BioLegend: 
468 TS1/18 (anti-CD18, PE/Cy7-conjugated), ICRF44 (anti-CD11b, BV421-conjugated), 469 5E8/CXCR2 (anti-CD182, APC-conjugated), CC2C6 (anti-CD47, PE/Cy7-conjugated), HA58 470 (anti-CD54, APC-conjugated), G10F5 (anti-CD66b, Pacific Blue-conjugated). PMN were 471 blocked with $2 \%$ normal mouse serum (Thermo Fisher Scientific) for 15 min at $4 \square$ C. After 472 washing, cells were resuspended in FACS buffer (PBS containing 0.5\% BSA and 2 mM EDTA;

473 all Sigma-Aldrich) and $100 \mu$ of equal number of cells were dispensed in several tubes and 474 stained with antibodies for $30 \mathrm{~min}$ at $4 \sqcap \mathrm{C}$. Antibodies were used diluted 1:2 $-1: 1,000$; optional 475 amount was determined by in-house titration. Cells were washed in FACS buffer and either 476 analyzed or fixed in $4 \%$ PFA for $15 \mathrm{~min}$ at $4 \square \mathrm{C}$ and analyzed the next day. Marker expression 477 was analyzed in a Guava 8HT using Guava ExpressPro software (Luminex, USA) or BD LSRII 478 using FACSDiva software (BD Biosciences, USA) and analyzed with FlowJo software (v10, 479 Tree Star).

\section{Cytokine and chemokine measurements}

482 Cytokines and chemokines were measured by electrochemiluminescence microarray using 483 commercial assays (Meso Scale Diagnostic, USA) following the manufacturer's instructions. 484 IFN- $\gamma$, IL-1 $\beta$, IL-6, IL-10, IL-12p70, TNF- $\alpha$, MCP-1, TGF- $\beta 1$, and IL-8 were reported as total 485 amount $(\mathrm{pg})$ contained in the entire volume of apical $(100 \mu \mathrm{l})$ and basolateral $(600 \mu \mathrm{l})$ culture 486 supernatants.

\section{Statistical analysis}

489 Statistical significances were calculated using the Student's $t$-test unpaired, one-way or two-way 490 analysis of variance (ANOVA) with Tukey's post-test as appropriate. PCA was performed by 491 selecting PC with eigenvalues greater than 1.0 (Kaiser rule). Plots and statistical tests were 492 performed using Prism software v9 (GraphPad, San Diego, CA). Treatment comparisons 
493 included at least three replicates and three independent experiments. Differences were

494 considered statistically significant at $p$-value $\leq 0.05$. Exact $p$ values are indicated in each Figure.

495 Results are expressed as mean \pm standard error of mean (sem).

497 Acknowledgements

498 The authors want to thank Dr. Mark Donowitz, for critical reading of the manuscript and Robin

499 Barnes and Nancy Greenberg, Clinical Research Nurses at University of Maryland, for their

500 assistance in obtaining peripheral blood samples. The authors would like to acknowledge the

501 Integrated Physiology Core of the Hopkins Conte Digestive Disease Basic and Translational

502 Research Core Center (NIH P30 DK-089502 to N.C.Z.) for providing human enteroids and 503 growth factor conditioned media and the University of Maryland School of Medicine Center for

504 Innovative Biomedical Resources, Flow Cytometry Shared Service. This work was supported by 505 NIH/NIAID P01Al125181 Immunology and Enteroid Cores, and 1R01Al117734-01 to M.F.P.

\section{Author Contributions}

508 J.M.L-D. designed and performed experiments, conducted data analyses and interpretation.

509 M.D. provided initial assistance with enteroid monolayers. N.C.Z. and M.F.P. participated in 510 experimental design, data analyses and interpretation, and supervised the study. J.M.L-D. and 511 M.F.P. wrote the manuscript. All authors edited the manuscript.

\section{Disclosures}

514 The authors have no financial conflicts of interest. 


\section{References}

1. Peterson LW, Artis D. 2014. Intestinal epithelial cells: regulators of barrier function and immune homeostasis. Nat Rev Immunol 14:141-53.

2. Mestas J, Hughes CC. 2004. Of mice and not men: differences between mouse and human immunology. J Immunol 172:2731-8.

3. Gibbons DL, Spencer J. 2011. Mouse and human intestinal immunity: same ballpark, different players; different rules, same score. Mucosal Immunol 4:148-57.

4. Barker N, van Es JH, Kuipers J, Kujala P, van den Born M, Cozijnsen M, Haegebarth A, Korving J, Begthel $\mathrm{H}$, Peters PJ, Clevers H. 2007. Identification of stem cells in small intestine and colon by marker gene Lgr5. Nature 449:1003-7.

5. Zhang YG, Wu S, Xia Y, Sun J. 2014. Salmonella-infected crypt-derived intestinal organoid culture system for host-bacterial interactions. Physiol Rep 2:e12147.

6. Huang JY, Sweeney EG, Sigal M, Zhang HC, Remington SJ, Cantrell MA, Kuo CJ, Guillemin K, Amieva MR. 2015. Chemodetection and destruction of host urea allows Helicobacter pylori to locate the epithelium. Cell Host Microbe 18:147-56.

7. Co JY, Margalef-Catala M, Li X, Mah AT, Kuo CJ, Monack DM, Amieva MR. 2019. Controlling epithelial polarity: A human enteroid model for host-pathogen interactions. Cell Rep 26:2509-2520.e4.

8. Foulke-Abel J, In J, Kovbasnjuk O, Zachos NC, Ettayebi K, Blutt SE, Hyser JM, Zeng XL, Crawford SE, Broughman JR, Estes MK, Donowitz M. 2014. Human enteroids as an ex-vivo model of host-pathogen interactions in the gastrointestinal tract. Exp Biol Med (Maywood) 239:1124-34.

9. In J, Foulke-Abel J, Zachos NC, Hansen AM, Kaper JB, Bernstein HD, Halushka M, Blutt S, Estes MK, Donowitz M, Kovbasnjuk O. 2016. Enterohemorrhagic Escherichia coli reduce mucus and intermicrovillar bridges in human stem cell-derived colonoids. Cell Mol Gastroenterol Hepatol 2:48-62 e3.

10. Rajan A, Vela L, Zeng XL, Yu X, Shroyer N, Blutt SE, Poole NM, Carlin LG, Nataro JP, Estes MK, Okhuysen PC, Maresso AW. 2018. Novel segment- and host-specific patterns of Enteroaggregative Escherichia coli adherence to human intestinal enteroids. mBio 9:e02419-17.

11. Noel G, Baetz NW, Staab JF, Donowitz M, Kovbasnjuk O, Pasetti MF, Zachos NC. 2017. A primary human macrophage-enteroid co-culture model to investigate mucosal gut physiology and host-pathogen interactions. Sci Rep 7:45270.

12. In JG, Foulke-Abel J, Estes MK, Zachos NC, Kovbasnjuk O, Donowitz M. 2016. Human mini-guts: new insights into intestinal physiology and host-pathogen interactions. Nat Rev Gastroenterol Hepatol 13:633-642.

13. Noel G, Doucet M, Nataro JP, Kaper JB, Zachos NC, Pasetti MF. 2018. Enterotoxigenic Escherichia coli is phagocytosed by macrophages underlying villus-like intestinal epithelial cells: modeling ex vivo innate immune defenses of the human gut. Gut Microbes 9:382-389.

14. Sansonetti PJ, Arondel J, Cantey JR, Prevost MC, Huerre M. 1996. Infection of rabbit Peyer's patches by Shigella flexneri: effect of adhesive or invasive bacterial phenotypes on follicle-associated epithelium. Infect Immun 64:2752-64.

15. Gustafson MP, Lin Y, Maas ML, Van Keulen VP, Johnston PB, Peikert T, Gastineau DA, Dietz AB. 2015. A method for identification and analysis of non-overlapping myeloid immunophenotypes in humans. PLoS One 10:e0121546.

16. Metzemaekers M, Vandendriessche S, Berghmans N, Gouwy M, Proost P. 2020. Truncation of CXCL8 to CXCL8(9-77) enhances actin polymerization and in vivo migration of neutrophils. J Leukoc Biol 107:1167-1173.

17. Fournier BM, Parkos CA. 2012. The role of neutrophils during intestinal inflammation. Mucosal Immunol 5:354-66. 
18. Lekstrom-Himes JA, Gallin JI. 2000. Immunodeficiency diseases caused by defects in

19. phagocytes. N Engl J Med 343:1703-14.

19. Wortmann SB, Van Hove JLK, Derks TGJ, Chevalier N, Knight V, Koller A, Oussuren E, Mayr JA, van Spronsen FJ, Lagler FB, Gaughan S, Van Schaftingen E, Veiga-da-Cunha M. 2020. Treating neutropenia and neutrophil dysfunction in glycogen storage disease IB with an SGLT2-inhibitor. Blood doi:10.1182/blood.2019004465:2019004465.

20. Blikslager AT, Moeser AJ, Gookin JL, Jones SL, Odle J. 2007. Restoration of barrier function in injured intestinal mucosa. Physiol Rev 87:545-64.

21. Sullivan DP, Bui T, Muller WA, Butin-Israeli V, Sumagin R. 2018. In vivo imaging reveals unique neutrophil transendothelial migration patterns in inflamed intestines. Mucosal Immunol 11:1571-1581.

22. Lammermann T, Afonso PV, Angermann BR, Wang JM, Kastenmuller W, Parent CA, Germain RN. 2013. Neutrophil swarms require LTB4 and integrins at sites of cell death in vivo. Nature 498:371-5.

23. Uderhardt S, Martins AJ, Tsang JS, Lammermann T, Germain RN. 2019. Resident macrophages cloak tissue microlesions to prevent neutrophil-driven inflammatory damage. Cell 177:541-555 e17.

24. Sumagin R, Robin AZ, Nusrat A, Parkos CA. 2014. Transmigrated neutrophils in the intestinal lumen engage ICAM-1 to regulate the epithelial barrier and neutrophil recruitment. Mucosal Immunol 7:905-15.

25. Parkos CA, Delp C, Arnaout MA, Madara JL. 1991. Neutrophil migration across a cultured intestinal epithelium. Dependence on a CD11b/CD18-mediated event and enhanced efficiency in physiological direction. J Clin Invest 88:1605-12.

26. Brazil JC, Liu R, Sumagin R, Kolegraff KN, Nusrat A, Cummings RD, Parkos CA, Louis NA. 2013. alpha3/4 Fucosyltransferase 3-dependent synthesis of Sialyl Lewis A on CD44 variant containing exon 6 mediates polymorphonuclear leukocyte detachment from intestinal epithelium during transepithelial migration. J Immunol 191:4804-17.

27. Flemming S, Luissint AC, Nusrat A, Parkos CA. 2018. Analysis of leukocyte transepithelial migration using an in vivo murine colonic loop model. JCl Insight 3:e99722.

28. Masoodi I, Kochhar R, Dutta U, Vaishnavi C, Prasad KK, Vaiphei K, Hussain S, Singh K. 2012. Evaluation of fecal myeloperoxidase as a biomarker of disease activity and severity in ulcerative colitis. Dig Dis Sci 57:1336-40.

29. Kolaczkowska E, Kubes P. 2013. Neutrophil recruitment and function in health and inflammation. Nat Rev Immunol 13:159-75.

30. Grunwell JR, Giacalone VD, Stephenson S, Margaroli C, Dobosh BS, Brown MR, Fitzpatrick AM, Tirouvanziam R. 2019. Neutrophil dysfunction in the airways of children with acute respiratory failure due to lower respiratory tract viral and bacterial coinfections. Sci Rep 9:2874.

31. Svedberg FR, Brown SL, Krauss MZ, Campbell L, Sharpe C, Clausen M, Howell GJ, Clark H, Madsen J, Evans CM, Sutherland TE, Ivens AC, Thornton DJ, Grencis RK, Hussell T, Cunoosamy DM, Cook PC, MacDonald AS. 2019. The lung environment controls alveolar macrophage metabolism and responsiveness in type 2 inflammation. Nat Immunol 20:571-580.

32. Vainer B, Nielsen OH, Horn T. 2000. Comparative studies of the colonic in situ expression of intercellular adhesion molecules (ICAM-1, -2, and -3), beta2 integrins (LFA-1, Mac-1, and p150,95), and PECAM-1 in ulcerative colitis and Crohn's disease. Am J Surg Pathol 24:1115-24.

33. Liu Y, Merlin D, Burst SL, Pochet M, Madara JL, Parkos CA. 2001. The role of CD47 in neutrophil transmigration. Increased rate of migration correlates with increased cell surface expression of CD47. J Biol Chem 276:40156-66. 
618

619

620

621

622

623

624

625

626

627

628

629

630

631

632

633

634

635

636

637

638

639

640

641

642

643

644

645

646

647

648

649

650

651

652

653

654

655

656

657

658

659

660

661

662

663

664

665

666

667

668

34. Parkos CA, Colgan SP, Liang TW, Nusrat A, Bacarra AE, Carnes DK, Madara JL. 1996. CD47 mediates post-adhesive events required for neutrophil migration across polarized intestinal epithelia. J Cell Biol 132:437-50.

35. Golay J, Valgardsdottir R, Musaraj G, Giupponi D, Spinelli O, Introna M. 2019. Human neutrophils express low levels of FcgammaRIIIA, which plays a role in PMN activation. Blood 133:1395-1405.

36. Teschner D, Cholaszczynska A, Ries F, Beckert H, Theobald M, Grabbe S, Radsak M, Bros M. 2019. CD11b regulates fungal outgrowth but not neutrophil recruitment in a mouse model of invasive pulmonary aspergillosis. Front Immunol 10:123.

37. Zhao L, Xu S, Fjaertoft G, Pauksen K, Hakansson L, Venge P. 2004. An enzyme-linked immunosorbent assay for human carcinoembryonic antigen-related cell adhesion molecule 8, a biological marker of granulocyte activities in vivo. J Immunol Methods 293:207-14.

38. Altstaedt J, Kirchner H, Rink L. 1996. Cytokine production of neutrophils is limited to interleukin-8. Immunology 89:563-8.

39. Andrews C, McLean MH, Durum SK. 2018. Cytokine tuning of intestinal epithelial function. Front Immunol 9:1270.

40. Ruder B, Atreya R, Becker C. 2019. Tumour necrosis factor alpha in intestinal homeostasis and gut related diseases. Int J Mol Sci 20.

41. Reinecker HC, Loh EY, Ringler DJ, Mehta A, Rombeau JL, MacDermott RP. 1995. Monocyte-chemoattractant protein 1 gene expression in intestinal epithelial cells and inflammatory bowel disease mucosa. Gastroenterology 108:40-50.

42. Raqib R, Lindberg AA, Wretlind B, Bardhan PK, Andersson U, Andersson J. 1995. Persistence of local cytokine production in shigellosis in acute and convalescent stages. Infect Immun 63:289-96.

43. Kotloff KL, Riddle MS, Platts-Mills JA, Pavlinac P, Zaidi AKM. 2018. Shigellosis. Lancet 391:801-812.

44. Ranganathan S, Doucet M, Grassel CL, Delaine-Elias B, Zachos NC, Barry EM. 2019. Evaluating Shigella flexneri pathogenesis in the human enteroid model. Infect Immun 87:e00740-18.

45. Koestler BJ, Ward CM, Fisher CR, Rajan A, Maresso AW, Payne SM. 2019. Human intestinal enteroids as a model system of Shigella pathogenesis. Infect Immun 87:e00733-18.

46. Perdomo OJ, Cavaillon JM, Huerre M, Ohayon H, Gounon P, Sansonetti PJ. 1994. Acute inflammation causes epithelial invasion and mucosal destruction in experimental shigellosis. J Exp Med 180:1307-19.

47. Perdomo JJ, Gounon P, Sansonetti PJ. 1994. Polymorphonuclear leukocyte transmigration promotes invasion of colonic epithelial monolayer by Shigella flexneri. $\mathrm{J}$ Clin Invest 93:633-43.

48. Beatty WL, Sansonetti PJ. 1997. Role of lipopolysaccharide in signaling to subepithelial polymorphonuclear leukocytes. Infect Immun 65:4395-404.

49. Yardley JH, Donowitz M. 1977. Colo-rectal biopsy in inflammatory bowel disease. Monogr Pathol:50-94.

50. Coron E, Flamant M, Aubert P, Wedel T, Pedron T, Letessier E, Galmiche JP, Sansonetti PJ, Neunlist M. 2009. Characterisation of early mucosal and neuronal lesions following Shigella flexneri infection in human colon. PLoS One 4:e4713.

51. Kuhn KA, Manieri NA, Liu TC, Stappenbeck TS. 2014. IL-6 stimulates intestinal epithelial proliferation and repair after injury. PLoS One 9:e114195.

52. Ye D, Ma I, Ma TY. 2006. Molecular mechanism of tumor necrosis factor-alpha modulation of intestinal epithelial tight junction barrier. Am J Physiol Gastrointest Liver Physiol 290:G496-504. 
53. Sellge G, Magalhaes JG, Konradt C, Fritz JH, Salgado-Pabon W, Eberl G, Bandeira A, Di Santo JP, Sansonetti PJ, Phalipon A. 2010. Th17 cells are the dominant T cell subtype primed by Shigella flexneri mediating protective immunity. J Immunol 184:207685.

54. Lindberg FP, Bullard DC, Caver TE, Gresham HD, Beaudet AL, Brown EJ. 1996. Decreased resistance to bacterial infection and granulocyte defects in IAP-deficient mice. Science 274:795-8.

55. Azcutia V, Kelm M, Luissint AC, Boerner K, Flemming S, Quiros M, Newton G, Nusrat A, Luscinskas FW, Parkos CA. 2020. Neutrophil expressed CD47 regulates CD11b/CD18dependent neutrophil transepithelial migration in the intestine in vivo. Mucosal Immunol doi:10.1038/s41385-020-0316-4.

56. Jaiswal $\mathrm{S}$, Jamieson $\mathrm{CH}$, Pang WW, Park CY, Chao MP, Majeti R, Traver D, van Rooijen N, Weissman IL. 2009. CD47 is upregulated on circulating hematopoietic stem cells and leukemia cells to avoid phagocytosis. Cell 138:271-85.

57. Zhang Z, Jin L, Champion G, Seydel KB, Stanley SL, Jr. 2001. Shigella infection in a SCID mouse-human intestinal xenograft model: role for neutrophils in containing bacterial dissemination in human intestine. Infect Immun 69:3240-7.

58. Staab JF, Lemme-Dumit JM, Latanich R, Pasetti MF, Zachos NC. 2020. Co-Culture System of Human Enteroids/Colonoids with Innate Immune Cells. Curr Protoc Immunol $131: e 113$.

59. Sato T, Stange DE, Ferrante M, Vries RG, Van Es JH, Van den Brink S, Van Houdt WJ, Pronk A, Van Gorp J, Siersema PD, Clevers H. 2011. Long-term expansion of epithelial organoids from human colon, adenoma, adenocarcinoma, and Barrett's epithelium. Gastroenterology 141:1762-72.

60. Parkos CA, Colgan SP, Delp C, Arnaout MA, Madara JL. 1992. Neutrophil migration across a cultured epithelial monolayer elicits a biphasic resistance response representing sequential effects on transcellular and paracellular pathways. J Cell Biol 117:757-64.

61. Brouwer N, Dolman KM, van Houdt M, Sta M, Roos D, Kuijpers TW. 2008. Mannosebinding lectin (MBL) facilitates opsonophagocytosis of yeasts but not of bacteria despite MBL binding. J Immunol 180:4124-32.

62. Chaka W, Scharringa J, Verheul AF, Verhoef J, Van Strijp AG, Hoepelman IM. 1995. Quantitative analysis of phagocytosis and killing of Cryptococcus neoformans by human peripheral blood mononuclear cells by flow cytometry. Clin Diagn Lab Immunol 2:753-9. 


\section{Figure legends}

705 Figure 1. Establishment of a human PMN-enteroid co-culture. (A) May-Grünwald Giemsa

706 image of PMN isolated from peripheral blood [left]. Representative dot plot of PMN phenotype

707 [middle] and schematic representation the PMN-enteroid co-culture model [right]. (B) Confocal

$708 \mathrm{XZ}$ projection [top; right, magnification $\mathrm{XY}$ projection] and $\mathrm{H} \& \mathrm{E}$ [bottom] images of the PMN-

709 enteroid co-culture. Confocal: DNA, blue; actin, white; CD16 (PMN), green; Transwell insert,

710 dashed lines. Arrowheads indicate PMN seeded on the Transwell insert and at the base of the

711 columnar epithelium. (C) Representative immunofluorescence confocal microscopy image [top,

$712 \mathrm{XY}$; bottom, $\mathrm{XZ}$ projections] of the enteroid apical surface showing PMN intercalated and

713 moving up within the epithelial cell monolayer. DNA, blue; CD16 (PMN), green; actin, white;

714 Transwell insert, dashed line. (D) Number of PMN transmigrated to the luminal compartment $2 \mathrm{~h}$

715 after being added to the co-culture in the absence or presence of apically delivered rhlL-8. (E)

716 TER of enteroid monolayers and PMN-enteroid co-cultures in a $2 \mathrm{~h}$ co-culture. (F) TER of

717 enteroid monolayers and PMN-enteroid co-culture apically treated with rhlL-8 for 2h. (G) Total

718 amount of cytokines secreted into the apical and basolateral compartments after $2 \mathrm{~h}$ of co-

719 culture. (D, E, F) Each dot represents the average from three replicate wells; data are shown as

720 mean \pm sem from 3 independent experiments. (G) Data are shown as mean + sem from 3

721 independent experiments in triplicate. $p$ values were calculated by Student's $t$-test. See also

722 Figure S1.

723

724 Figure 2. Distinctive phenotype of PMN within the human intestinal environment. (A)

725 Schematic representation of PMN in direct contact with epithelial cells (C) or in the basolateral

726 media (BM). (B, C, D) Phenotype of isolated PMN, PMN in C and BM determined by flow

727 cytometry within $2 \mathrm{~h}$ of co-culture. Each dot represents data collected from three replicate wells;

728 data are shown as mean \pm sem from 3 independent experiments. $p$ values were calculated by

729 one-way-ANOVA with Tukey's post-test for multiple comparisons. See also Figure S2. 
731 Figure 3. Innate immune responses of human PMN to S. flexneri. (A) Representative

732 confocal microscopy image and histogram of $S$. flexneri 2a-FITC uptake by human PMN.

733 Confocal image [left, XY projection]. DNA, blue; actin, red; S. flexneri 2a, green. Histograms of

734 PMN harboring Shigella-FITC [middle] and percentage of phagocytosis at 10, 30 and 60 min

735 post infection [left]. Each dot represents the average from three replicates and PMN from 5

736 individual donors; data are shown as mean \pm sem from 3 independent experiments. (B)

737 Extracellular S. flexneri 2a colony forming units (CFU) in culture media following PMN exposure

738 to $S$. flexneri $2 \mathrm{a}\left(1 \times 10^{8} \mathrm{CFU} / \mathrm{ml}\right)$ for 30 and $60 \mathrm{~min}$; controls included $S$. flexneri 2 a in culture

739 media or in PMN-conditioned media without PMN. Each dot represents the average from three

740 replicates and PMN from 6 individual donors; data are shown as mean \pm sem from 3

741 independent experiments. (A, B) $p$ values were calculated by one-way ANOVA with Tukey's

742 post-test for multiple comparisons. (C) PMN viability in the presence and absence of S. flexneri

743 2a. Data represents the average from three replicates and PMN from 3 individual donors; data

744 are shown as mean \pm sem from 3 independent experiments. (D) PMN morphology before $(0$

$745 \mathrm{~min}$ ) and after (30 $\mathrm{min})$ exposure to $S$. flexneri 2a. (E) Confocal microscopy image [XY

746 projection] of NETs 30 min after exposure to S. flexneri 2a. DNA, blue; actin, red; S. flexneri 2a,

747 green. (F) Immunofluorescence image showing S. flexneri 2a-FITC colocalization in PMN

748 lysosome. Arrowheads indicate bacteria intracellularly and within the lysosome compartment.

749 (G) Total amount of cytokines secreted in culture supernatant of PMN alone and PMN exposed

750 to $S$. flexneri 2 a for $2 \mathrm{~h}$. Data are shown as mean \pm sem from 3 independent experiments in

751 triplicate. $(\mathrm{C}, \mathrm{G}) p$ values were calculated by Student's $t$ test. $(\mathbf{H})$ Principal component analysis

752 (PCA) plot of Shigella-exposure PMN v. PMN monoculture. Variables analyzed: phagocytosis,

753 bactericidal activity, viability, IL-1 $\beta, \mathrm{IL}-6, \mathrm{IL}-8, \mathrm{TNF}-\alpha$, and MCP-1 variables. 

cells and PMN. (A) Numbers of PMN transmigrated to the apical compartment of enteroid

757 monolayers exposed or not to $S$. flexneri 2a for $2 \mathrm{~h}$. (B and C) Confocal microscopy images [B:

758 top, $\mathrm{XY}$; bottom, $\mathrm{XZ}$ projections; right, magnification] of PMN-enteroid co-culture infected with $S$.

759 flexneri 2a for 2h. PMN: CD16 (B, green), CD15 (C, red); S. flexneri 2a (B, red; C, green); actin,

760 white; nuclei, blue. White arrowheads indicate S. flexneri 2a. (D) CFU in the apical and

761 basolateral media of enteroid and PMN-enteroid co-cultures apically exposed to $S$. flexneri $2 a$

762 for $2 \mathrm{~h}$. (E) TER of enteroid and PMN-enteroid co-cultures exposed to S. flexneri 2a for $2 \mathrm{~h}$. (F)

763 Total amount of cytokines in the apical and basolateral media of PMN-enteroid co-cultures

764 exposed or not to $S$. flexneri 2a for $2 \mathrm{~h}$. (A, D, E) Each dot represents the average from three

765 replicate wells; data are shown as mean \pm sem from $3(A, E)$ and 5 (D) independent

766 experiments. $p$ values were calculated by Student's $t$-test. (F) Data are shown as mean \pm sem

767 from 3 independent experiments in triplicate. $p$ values were calculated by Student's $t$-test.

769 Figure 5. Immune phenotype of PMN co-cultured with epithelial cells and exposed to

770 Shigella. (A, B, C) Cell surface expression of CD15, CD16, CD11b, CD18, CD88, CD66b,

771 CD47, and CD182 on PMN embedded in the enteroid monolayer (co-culture) exposed or not to

772 S. flexneri $2 \mathrm{a}$ for $2 \mathrm{~h}$. Each dot represents data collected from three replicate wells; data are

773 shown as mean \pm sem from 3 independent experiments. $p$ values were calculated by Student's

$774 \quad t$-test. See also Figure S3. 
EioRyiv preprint doi: https://doi.org/10.1101/2020.09.03.281535; this version posted March 20, 2021. The copyright holder for this preprint 1901 (which was not certified by peer review) is the author/funder. All rights reserved. No reuse allowed without permission.

\section{A}

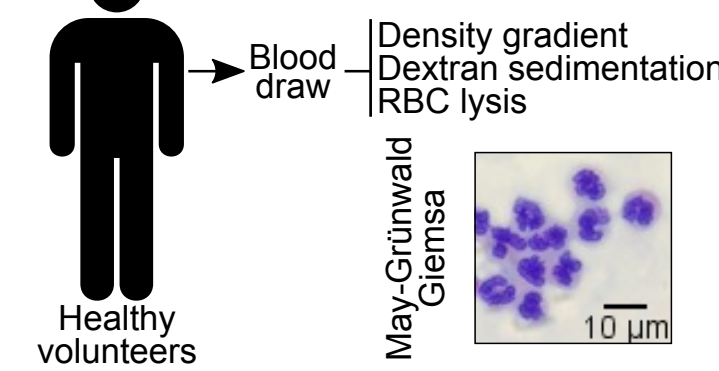

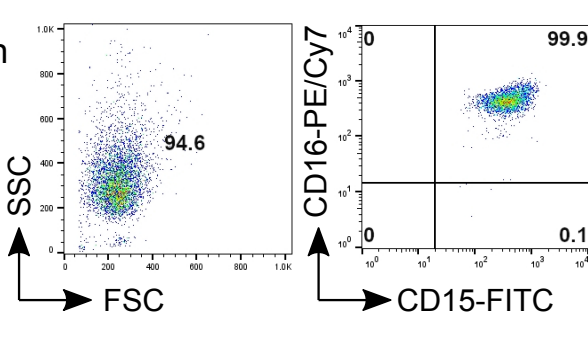

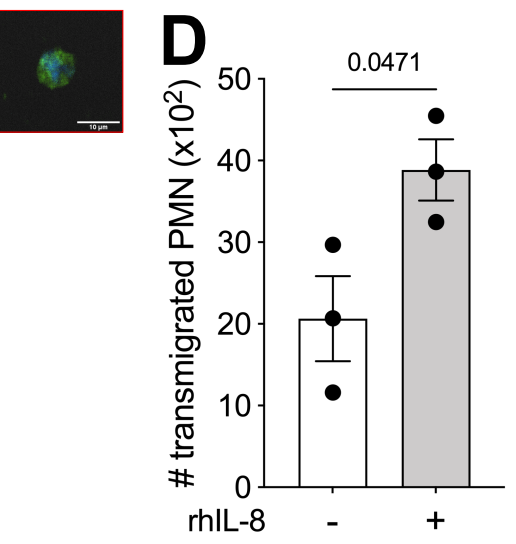

G

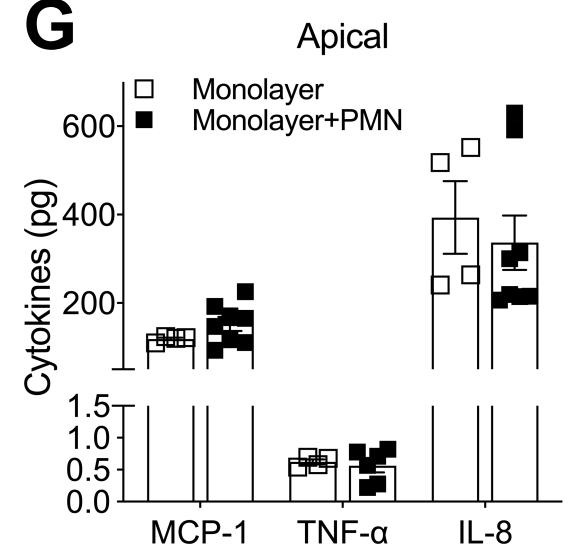

Apical
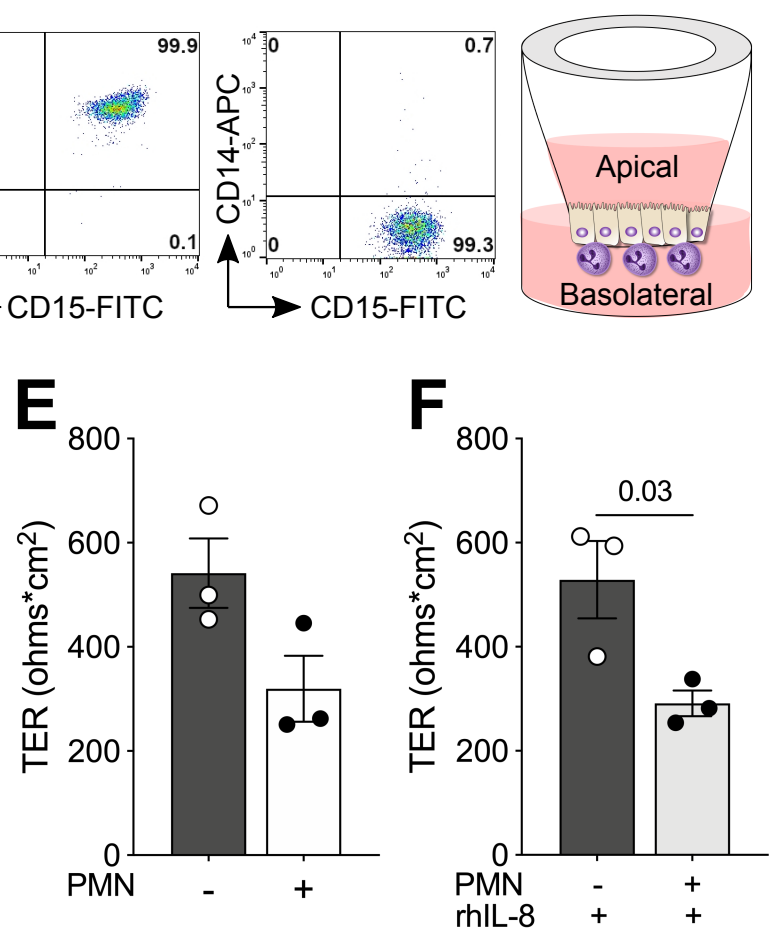

Basolateral

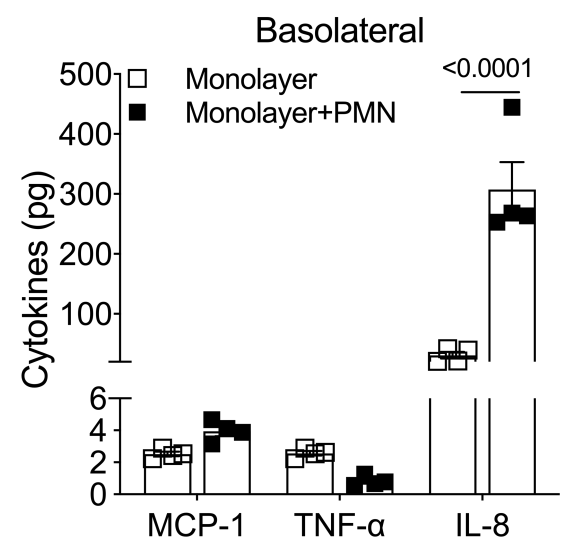

\section{C}
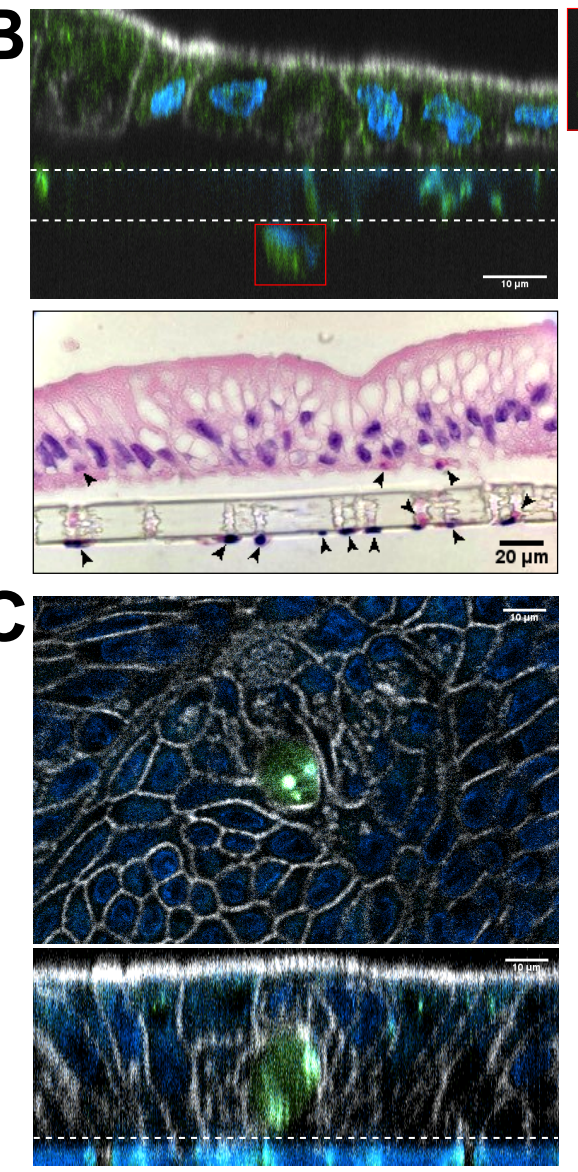
EH biolajv preprint doi: https://doi.org/10.1101/2020.09.03.281535; this version posted March 20, 2021. The copyright holder for this preprint - 9 Ure (which was not certified by peer review) is the author/funder. All rights reserved. No reuse allowed without permission.

A
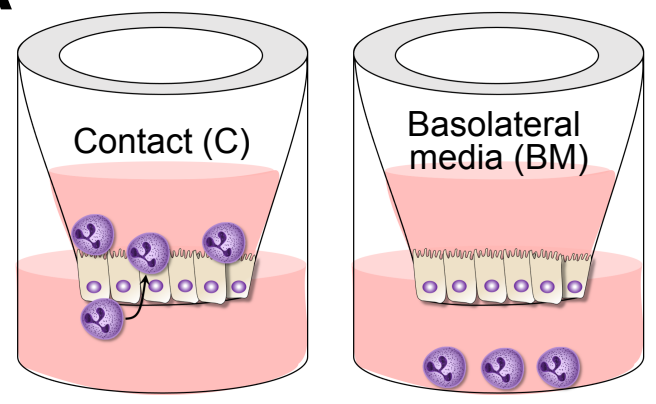

$\mathrm{C}_{1}$

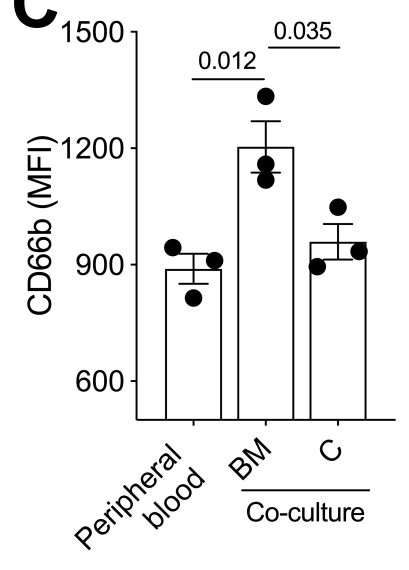

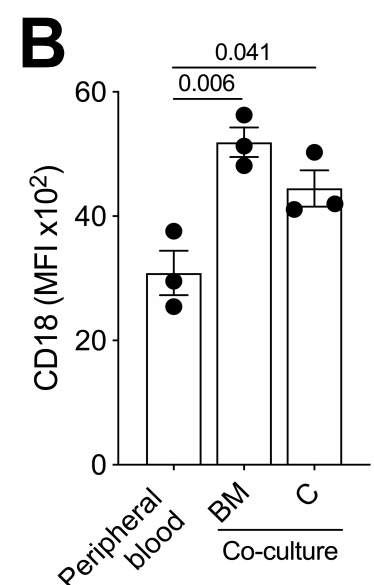

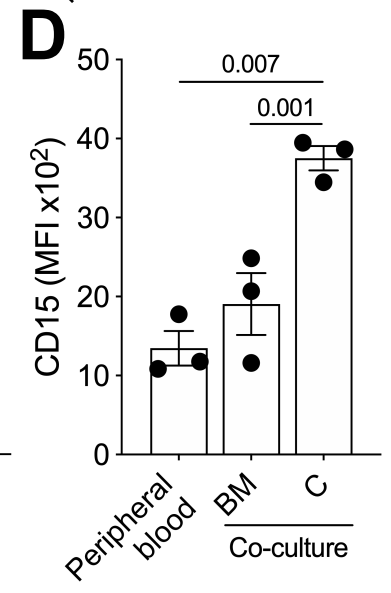

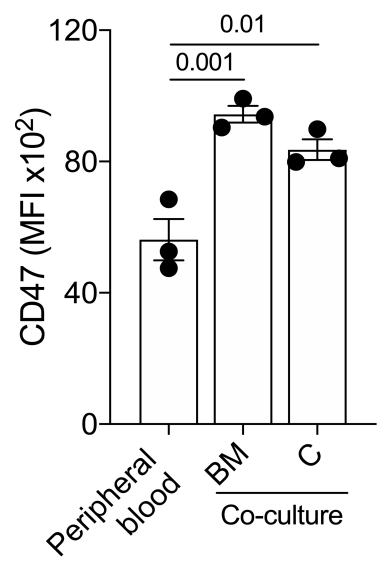
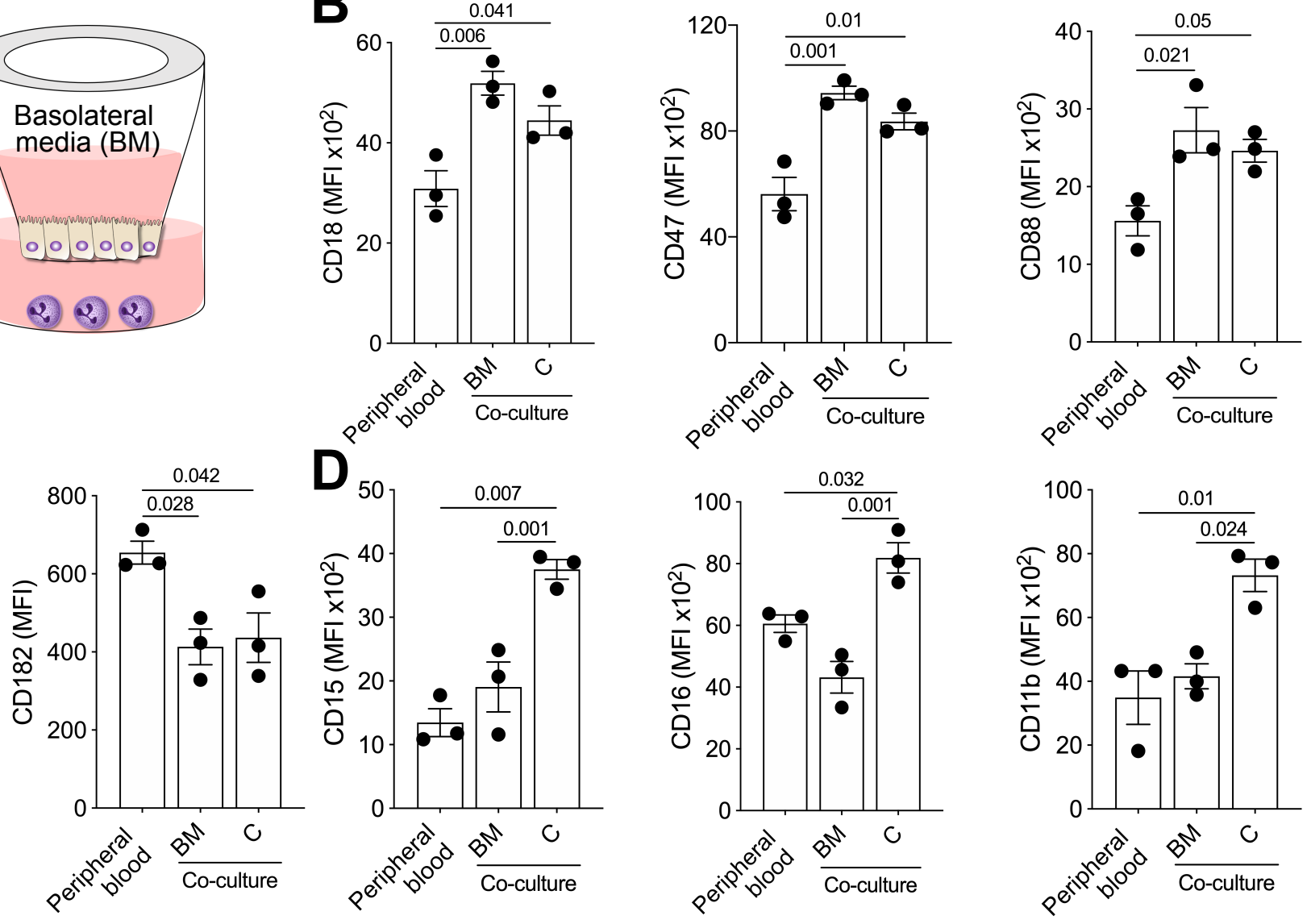
E bioRyiv preprint doi: https://doi.org/10.1101/2020.09.03.281535; this version posted March 20, 2021. The copyright holder for this preprint (which was not certified by peer review) is the author/funder. All rights reserved. No reuse allowed without permission.
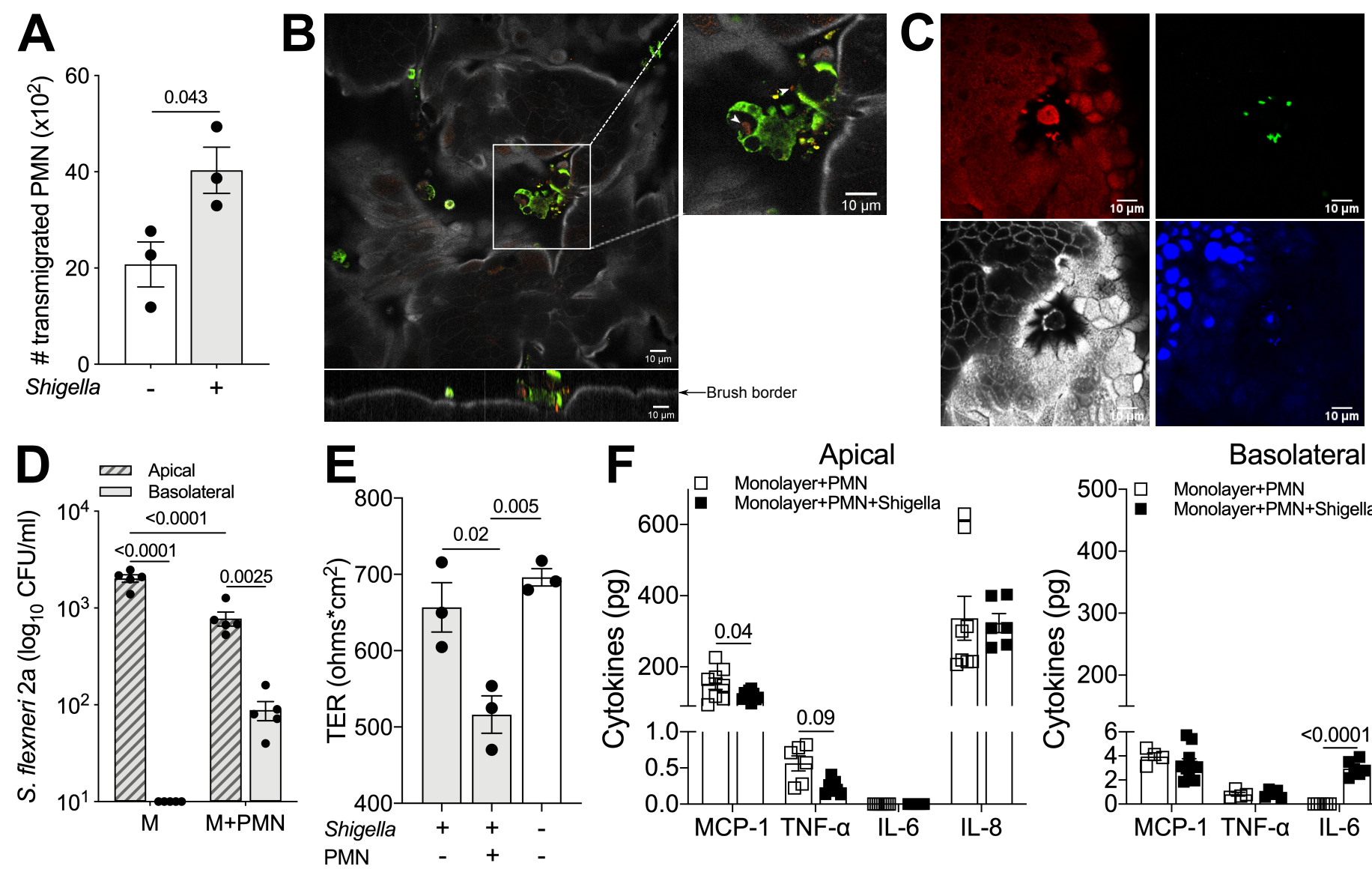

Basolateral

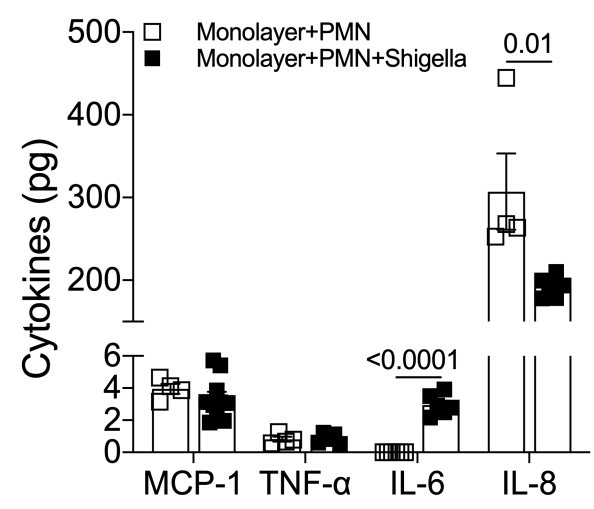


